\title{
Avances metodológicos para el análisis y la comprensión de la desventaja social femenina
}

\author{
M. ${ }^{a}$ Luz de la Cal Barredo \\ Universidad del País Vasco/Euskal Herriko Unibertsitatea \\ luz.cal@ehu.es
}

\begin{abstract}
Resumen
En las siguientes páginas pretendemos profundizar en el tratamiento dado a la cuestión del género en los análisis empíricos y teóricos referentes a la pobreza y la exclusión social. Para ello, justificaremos la pertinencia de incorporar la perspectiva de género al análisis de la desventaja social, analizando después las potencialidades y limitaciones de los diferentes enfoques sobre pobreza y exclusión social para estudiar el origen y las dimensiones de la desventaja social femenina. Por último, examinaremos bajo la óptica de género algunas estadísticas sobre pobreza y exclusión social, con el objetivo de mostrar los avances metodológicos presentes en algunas de ellas, y también las posibles lineas de mejora.

Palabras clave: pobreza, género, exclusión social, análisis cuantitativo.
\end{abstract}

\begin{abstract}
In the pages below we aim to focus on how the issue of gender is dealt with in empirical and theoretical analyses of poverty and social exclusion. We shall justify the relevance of including the gender perspective in our analysis of social disadvantage and then go on to analyze a variety of approaches to poverty and social exclusion, and study the origin and the dimension of the social disadvantage of females. Finally, we shall study, from the gender point of view, some statistics on poverty and social exclusion with the purpose of revealing not only the methodological advances that some of them show but also possible areas of improvement.
\end{abstract}

Key words: poverty, gender, social exclusion, quantitative analysis. 


\section{Justificación de la necesidad de un enfoque sensible al género en el estudio de la pobreza y la exclusión social}

En este primer epígrafe desarrollaremos tres líneas argumentales para justificar la necesidad de contar con un enfoque sensible al género a la hora de estudiar la pobreza y la exclusión social: la primera tiene que ver con las relaciones entre la definición de estos problemas y las políticas implementadas para solucionarlos; la segunda atiende a la desigual posición de mujeres y hombres en nuestras sociedades, lo que determina un acceso desigual a los recursos y al poder, y la tercera hace referencia a la mayor incidencia de la pobreza entre las mujeres.

En el cuerpo metodológico para el estudio de la pobreza existen cuatro temas fundamentales que están unidos: los conceptos, las definiciones operacionales (que incluyen las medidas), las explicaciones y las políticas. Los cuatro son de igual importancia y los avances en uno dependen de los avances en los otros. Ninguno de ellos puede ser tomado como punto de partida ni como analíticamente distinto de los otros. Las definiciones de pobreza dependen, así pues, de las políticas propuestas y, lo que es más importante, las políticas puestas en práctica para luchar contra la pobreza afectan a la definición previa de pobreza, dando lugar a una especie de argumento circular, un enigma del tipo «gallina-huevo» (Alcock, 1997).

Los desacuerdos que se producen en cuanto a la definición de la pobreza están estrechamente relacionados con los desacuerdos en cuanto a sus causas y a las soluciones para aliviarla. Por tanto, definición, medida, causa y solución están estrechamente vinculadas, y entender la pobreza requiere apreciar las interrelaciones que se dan entre ellos. Lo que entendemos por pobreza depende, en cierto grado, de lo que se intenta o espera hacer sobre ella, así que el debate académico y político sobre la pobreza no es sólo descriptivo, sino también prescriptivo, y la pobreza es inevitablemente un concepto político. Las definiciones de pobreza, por tanto, no son neutrales. Las personas son pobres porque no tienen los recursos necesarios para sobrevivir: la pobreza es un problema de escasez de recursos, un problema de naturaleza económica. Pero también tiene una dimensión política. Estudiar la pobreza lleva a plantearnos qué son los recursos, quién los produce y quién los controla. La investigación sobre pobreza exige una visión integral y holística, que abarque un cuestionamiento de los recursos de una sociedad, su producción y distribución (Monreal, 1996: 105-106).

Si nos aproximamos al estudio de la pobreza sin tener en cuenta que las mujeres y los hombres tienen atributos, responsabilidades, experiencias de vida, oportunidades e intereses diferentes, no seremos capaces de estimar la incidencia real de la pobreza en una población, ni mucho menos conocer los principales factores que la provocan. Así mismo, las estrategias que propongamos para hacer frente a la pobreza resultarán 
ineficaces para una parte de la población. Y lo que es más importante, si no se incorpora la perspectiva de género, las políticas de lucha contra la pobreza pueden llegar incluso a empeorar las condiciones de vida de las mujeres empobrecidas. La razón reside en que lo que se hace para el estado, la región, e incluso para el hogar, se distribuye con independencia de la desigual posición social de mujeres y hombres. Dado que éstas se encuentran en una posición de desventaja, pueden beneficiarse menos de las medidas adoptadas. De esta manera, las desigualdades, lejos de reducirse, se amplían.

Con la perspectiva de género se busca destacar las desigualdades que sufren las mujeres respecto a los hombres, y que constituyen un obstáculo para que puedan desarrollar una mejor calidad de vida, ampliar su autonomía y ejercer sus derechos de ciudadanía. Las dos principales desigualdades se refieren, por un lado, al acceso, el uso y el control de los recursos productivos (trabajo, tierra, capital, formación, información, nuevas tecnologías, recursos naturales y vivienda), y ello explica las limitaciones de las mujeres para acceder a un nivel de ingresos adecuado. Por otro lado, cabe destacar que las desigualdades en el acceso al poder determinan que las mujeres participen en menor medida que los hombres en los procesos de toma de decisiones económicas y políticas, tanto en el hogar como en la sociedad en general. Ello implica que los programas y políticas se diseñan sin tener en cuenta los intereses y el punto de vista de ellas.

Como ya es de sobra conocido, gran parte de esta posición de subordinación de las mujeres con respecto a los hombres tiene su origen en la división sexual del trabajo, que, durante largo tiempo, ha confinado a las mujeres a las tareas domésticas en el ámbito privado, donde han desarrollado un trabajo no remunerado y no reconocido. La limitación de espacios de participación para las mujeres supone unas menores oportunidades de acceso a recursos, lo que se pone de manifiesto en privaciones diversas que tienen que ver con el ámbito educativo, el laboral, el de los derechos sociales, etc. Ello tiene su expresión en las mayores tasas de analfabetismo de las mujeres comparadas con las de los hombres en amplias zonas del planeta, las todavía bajas tasas de ocupación y actividad femeninas que se dan incluso en economías con alto grado de desarrollo, las persistentes diferencias salariales entre hombres y mujeres, la mayor dependencia entre las mujeres de las prestaciones asistenciales, inferiores en cuantía a las contributivas y condicionadas a la situación familiar, etc.

Adoptando una perspectiva de género se reconoce que mujeres y hombres experimentan la pobreza de maneras diferentes y que «la probabilidad de ser pobres no se distribuye al azar entre la población» (Sen, 1998). Se trata de ver que las mujeres son pobres en tanto en cuanto esta situación está condicionada por el género; en otras palabras, la experiencia de la pobreza y la exclusión están condicionadas por las identidades de género. Esto significa que hombres y mujeres son definidos como seres humanos 
diferentes, cada uno de ellos con sus propias oportunidades, roles y responsabilidades (Brunet et alii, 2008: 80).

En cuanto a las cifras de pobreza entre las mujeres, hay que apuntar que casi cualquier estadística o estudio que consultemos, y sea cual sea el área geográfica analizada y/o la metodología de medición empleada, el resultado es un mayor número de mujeres pobres que de hombres, o una mayor incidencia y severidad de las situaciones de desventaja entre ellas.

En América Latina se observa un mayor número de mujeres pobres que de hombres pobres en la mayoría de los países, tanto en la zona rural como en la urbana. Además, esto se manifiesta con mayor intensidad entre las mujeres en edad activa (CEPAL, 2003). En EE.UU., en 2007, un 12,5\% de las personas se encontraba bajo el umbral de pobreza ${ }^{1}$. Entre las personas solas, la incidencia es más elevada (un 20\%), pero si se trata de mujeres lo es aún mas (22,2\%). Entre las familias monoparentales, la tasa de pobreza también suele ser superior a la media, pero con grandes diferencias en función del sexo de la persona de referencia. Si se trata de un hombre, la tasa es del $13,6 \%$, y si se trata de una mujer, asciende hasta el $28,3 \%$. Teniendo en cuenta que en ese año existían en EE.UU. unos 5 millones de familias monoparentales encabezadas por hombres y 14,5 millones de familias monoparentales encabezadas por mujeres, puede concluirse que existían muchas más mujeres pobres que hombres pobres (U.S. Census Bureau, 2008).

En la Unión Europea (UE), las diferencias en la incidencia de la pobreza entre hombres y mujeres no son tan intensas. Así, según datos del Panel de Hogares de la Unión Europea (PHOGUE) de 2001, las tasa de riesgo de pobreza antes de transferencias sociales en la UE de los 15 ascendía al 22\% entre los hombres y al 25\% entre las mujeres. En España, los datos de la última Encuesta de Condiciones de Vida (2007) revelan que, entre la población pobre, el 53,6\% son mujeres. En cuanto a la incidencia de la pobreza, es de un 18,6\% entre los hombres y de un 20,9\% entre las mujeres; además, la brecha entre estas dos tasas es mayor entre las personas de más edad ${ }^{2}$. En el País Vasco, según la Encuesta de Pobreza y Desigualdades Sociales de 2008, la tasa de pobreza grave de mantenimiento entre los hombres era del 3,3\%, mientras que entre las mujeres era del 8,2\%. En cuanto a las situaciones de falta de bienestar, tenían una incidencia del 18,5\% entre los hombres y del 34,7\% entre las mujeres.

El hecho de que en todos estos estudios se concluya que la pobreza acusa una mayor más incidencia entre las mujeres nos da idea de que no se trata de un fenómeno coyuntural o circunstancial, sino que puede ser tomado como un rasgo propio de nuestros

1 La estimación de la pobreza que se hace en EE.UU. es en términos absolutos, es decir, estableciendo el umbral de pobreza en un determinado nivel de renta que se considera el presupuesto mínimo necesario para no ser pobre.

2 Estas tasas de pobreza referentes a la UE y a España son relativas. En concreto, el umbral de pobreza se establece en el $60 \%$ del ingreso medio. 
sistemas, como algo consustancial a los mismos. Esta mayor incidencia o probabilidad de pobreza entre las mujeres, como ya hemos dicho antes, tiene que ver con la falta de reconocimiento, valoración y remuneración de las aportaciones que tradicionalmente han hecho las mujeres al sistema, esto es, las tareas que tienen que ver con el mantenimiento de la vida. Como esta aportación no se reconoce, o se reconoce de forma marginal, el acceso a los recursos es limitado o condicionado a las relaciones que se establezcan con los hombres, que son los que participan en la llamada esfera productiva.

En la década de 1990, los trabajos y estudios que se referían tanto a la feminización de la pobreza como a los hogares encabezados por mujeres como los más pobres entre los pobres, pusieron de manifiesto que las mujeres sufren desproporcionadamente la pobreza. Aunque estos dos enfoques han dado frutos innegables, puesto que han servido para dar visibilidad a las mujeres en las estadísticas y conseguir recursos para ellas en los programas sociales y de desarrollo, han sido también puestos en cuestión por convertirse en clichés para explicar las situaciones de todas las mujeres y en todos los contextos. Así, con respecto a la identificación de la pobreza femenina con los hogares encabezados por mujeres, algunas de sus consecuencias negativas serían que se da a entender que la pobreza femenina está confinada a estos hogares, lo cual no sólo no es cierto, sino que sirve para robustecer los discursos que presentan estos hogares como inferiores o distintos de la norma. Del mismo modo, aunque la tesis de la feminización de la pobreza ha sido una herramienta útil en pro de la equidad de género, es un concepto que entraña algunos riesgos, ya que vincula la pobreza con las mujeres y no con las relaciones de género, y reduce la desigualdad de género a una función de la pobreza. Esto puede suponer que las políticas e iniciativas se dirijan únicamente a las mujeres como individuos aislados, no a cambiar las relaciones desiguales de género (CHANT, 2003).

\section{Los enfoques para el estudio de la desventaja social: limitaciones y potencialidades para incorporar la perspectiva de género}

La pobreza no siempre se ha analizado desde una perspectiva de género. Antes de que las feministas contribuyeran al análisis, se consideraba que la población pobre estaba íntegramente formada por hombres, o bien se daba por sentado que las necesidades $\mathrm{e}$ intereses de las mujeres eran idénticos a los de los hombres jefes del hogar, y por ende podían supeditarse a ellos (Kabeer, 1998).

En las tres últimas décadas, la investigación y el activismo feminista han logrado el reconocimiento de la dimensión de género en el estudio de la pobreza. Además, el 
análisis de este complejo fenómeno ha ido ampliando sus fronteras desde los aspectos económicos y materiales hasta los que consideran las diferentes esferas de la vida y los componentes subjetivos ligados a la diversidad humana.

En este apartado haremos una revisión de los enfoques de pobreza más importantes y de sus principales potencialidades de cara a incorporar en ellos la perspectiva de género. Aunque en cualquiera de los enfoques se pueden introducir aspectos que mejoren su sensibilidad hacia el género, hay que tener en cuenta que los conceptos amplios de pobreza son más útiles que los enfoques estrechos y restringidos basados en los ingresos del hogar. La razón reside en que estos enfoques amplios permiten captar mejor aspectos multidimensionales de las desventajas relacionadas con el género, como son la falta de poder en la toma de decisiones en el hogar o la división sexual del trabajo y sus efectos sobre los medios materiales que consiguen las mujeres, así como sobre su propia identidad.

\subsection{El enfoque monetario}

Desde este enfoque, se establece una frontera monetaria (renta o gasto) y se considera pobre a quien tiene unos recursos insuficientes para alcanzar un nivel de vida mínimo para satisfacer las necesidades básicas. Se habla de pobreza económica o material. Es el enfoque de los economistas, al que se reprocha que reduce la pobreza a las carencias monetarias, sin tener en cuenta sus diversas dimensiones. Este es, sin duda, y valga la redundancia, el concepto de pobreza más pobre, ya que sólo hace referencia a los recursos materiales. Además, dificulta la incorporación de la perspectiva de género por varias razones (Clert, 1998):

Se centra en lo distributivo e individual, presentando la sociedad como una masa de individuos atomizados compitiendo en el mercado, lo que no ayuda a recoger la perspectiva relacional del enfoque de género.

No es un concepto activo que permita sugerir la responsabilidad de la acción pública o de la sociedad civil en la desventaja social. Nos presenta la pobreza, no como la responsabilidad de determinados mecanismos o actores sociales, sino como el resultado de operaciones impersonales e inevitables de las fuerzas económicas.

Pasando al problema de la cuantificación de la pobreza, cabe señalar que, frecuentemente, cuando se mide la pobreza bajo un enfoque monetario se omiten una serie de recursos que condicionan los niveles de bienestar de las personas y que, además, tienen una influencia importante en las cargas de pobreza que soportan mujeres y hombres ${ }^{3}$.

\footnotetext{
3 La problemática que presenta la medición monetaria de la pobreza es amplísima y no se refiere sólo a su falta de sensibilidad ante el género. Se trata de problemas relativos a cuáles son las necesidades que deben quedar satisfechas para no ser pobre, y a cuáles son los bienes que satisfacen tales necesidades; problemas con respecto a dónde establecer el umbral de pobreza, y problemas con respecto a la fiabilidad de los datos que proceden de las Encuestas de Presupuestos Familiares. A pesar de esto, la metodología para la medición de la pobreza ha sido y sigue siendo utilizada con
} 
Por lo común, los recursos que son tenidos en cuenta para evaluar la situación de pobreza o bienestar suelen restringirse a un concepto de renta bastante estrecho. Sin embargo, la renta no es la única dotación de recursos, sino un componente más, y no refleja las transferencias en especie ni la acumulación. Por ejemplo, los servicios públicos de salud o de educación, cuya influencia en el bienestar es crucial, no se suelen incluir.

Otro de los recursos que se deberían tener en cuenta para evaluar la situación del hogar es el capital social que se genera mediante las redes de parentesco, amistad y vecindad. Se trata de uno de los ejes que, junto con el mercado (esfera mercantil) y el de la protección social (esfera de la redistribución), están en la base de la integración social de las personas. La fortaleza en la redes de reciprocidad puede suplir debilidades en las otras dos esferas, proporcionado recursos monetarios o favoreciendo la participación en la esfera mercantil. Esto último resulta determinante en el caso de las mujeres con hijos a su cargo, cuya inserción laboral está condicionada a la existencia no sólo de servicios públicos de guardería o de escuela, sino también a apoyos procedentes de redes cercanas familiares, vecinales y de amistad. Estas redes también pueden proporcionar medios de subsistencia cuando fallan el empleo y la protección social.

Por último, en esta relación de omisiones a la hora evaluar la situación del hogar, debe señalarse la del trabajo doméstico y de cuidados, dada su centralidad en una perspectiva de género. En coherencia con una perspectiva que entiende la pobreza sólo como problema económico y que prioriza y valora exclusivamente la esfera mercantil, la medición de la pobreza basada en el enfoque monetario se lleva a cabo sin referencia alguna al trabajo doméstico y de cuidados. Sin embargo, la consideración del tiempo dedicado a estas tareas y su traducción en recursos monetarios es una manera de considerar lo que las mujeres aportan al bienestar del hogar. Otra forma sería calcular el coste en el que el hogar debería incurrir si tuviera que contratar esos servicios en el mercado. De esta manera, se evidenciarían aún más las diferencias de bienestar entre los hogares encabezados por hombres, en los que la mujer se dedica a estas tareas no remuneradas, y aquellos en los que hay que dedicar una parte de la renta a comprar estos servicios.

Otro de los obstáculos que presenta el enfoque monetario en cuanto a la perspectiva de género es que la unidad de análisis a la hora de cuantificar la pobreza es generalmente el hogar, entendido éste como unidad de residencia, de presupuesto y de gasto. La elección de esta unidad de análisis se justifica porque, al igual que en otros muchos comportamientos económicos y sociales, son los hogares los agentes económicos relevantes en gasto y consumo. Además, son unidades en las que las estrategias de los individuos para aprovisionarse de recursos están fuertemente interrelacionadas. Sin embargo, el tomar el hogar como unidad de análisis invisibiliza situaciones de pobreza

profusión, ya que presenta ventajas tales como su simplicidad, la familiaridad de la unidad de medida empleada y la disponibilidad de datos. 
potencial o vulnerabilidad en el caso de algunos colectivos, como la juventud no emancipada del hogar familiar, las personas de edad que cobran pensiones de jubilación insuficientes y las mujeres que no realizan trabajo remunerado. Por esto, cada vez más estudios e investigaciones ofrecen una cuantificación de la pobreza y la exclusión social referida tanto a los hogares como a las personas.

Algunas propuestas introducen la idea de que es necesario, a la hora de estudiar las situaciones de pobreza, introducir el concepto de autonomía económica. Se trataría de medir con qué ingresos cuenta cada persona del hogar para satisfacer sus necesidades. Esta medición, combinada con la del ingreso del hogar, puede servir para detectar situaciones de riesgo entre las mujeres. Se trataría de hogares no pobres, pero en los cuales la mujer no cuenta con ingresos propios, por lo que una ruptura conyugal o la viudedad podrían conllevar graves consecuencias económicas. Por otra parte, la medición de los ingresos a escala individual puede resultar especialmente esclarecedora para evaluar situaciones de pobreza si dentro del hogar los ingresos no se distribuyen adecuadamente de cara a satisfacer las necesidades de todos los componentes del hogar. Cuando medimos la pobreza por hogares estamos suponiendo que éstos son instituciones en los que no se dan problemas, ni desorganización, ni relaciones de poder, ni diferentes necesidades que satisfacer ${ }^{4}$. Sin embargo, sobre todo en determinados contextos, la dependencia, la falta de capacidad de decisión y de acceso a recursos que sufren las mujeres en numerosas instituciones de nuestra sociedad, puede producirse también en el espacio doméstico (CEPAL-UNIFEM, 2004: 20-21).

\subsection{El enfoque subjetivo/participativo}

Desde esta óptica, se considera que la pobreza tiene, sobre todo, una dimensión subjetiva y que la conciencia de la pobreza es un elemento constitutivo de la situación de pobreza. La pobreza se define a partir del análisis que las propias personas pobres hacen de su realidad. Las metodologías de este tipo poseen importantes ventajas a la hora de considerar el género en el estudio de la pobreza, porque de esta manera se incorpora cuál es la percepción del bienestar que tienen las propias personas afectadas por la pobreza, que no tiene por qué coincidir con la de la persona que investiga sobre la pobreza, que puede que ni sea pobre ni sea una mujer. En esta línea, cabe destacar el trabajo coordinado por Tortosa (2002) sobre mujeres pobres, en el que los investigadores ponen en suspenso sus propias visiones, valores y lo que forma parte de su bagaje cultural, y les dan la palabra a las mujeres a través de entrevistas en profundidad.

4 Estos dos aspectos, la estimación de las personas sin autonomía económica y la problemática del acceso no compartido a los recursos en el hogar, se tratan en el tercer epígrafe de este trabajo, en el que se hace referencia a la Encuesta de Pobreza y Desigualdades Sociales que se realiza en Euskadi. 
La incorporación de elementos subjetivos o participativos presenta la ventaja de que puede servir para contemplar aspectos del bienestar que van mucho más allá del ingreso, como son el control de los recursos, el acceso a los mismos, la pobreza de tiempo, la vulnerabilidad a la violencia doméstica ${ }^{5}$ la desigualdad en materia de toma de decisiones en el hogar. Estas metodologías también pueden ayudar a comprender mejor situaciones en las que las mujeres sufren amplias privaciones materiales como consecuencia de la separación o el divorcio, pero sin embargo se sienten mejor que anteriormente, porque tienen más poder para decidir sobre sus gastos, o para trabajar, o porque no tienen que soportar situaciones de violencia o de dependencia. Los análisis participativos han demostrado que mujeres y hombres tienen diferentes preocupaciones e intereses en cuanto a cuáles son las necesidades básicas o los servicios que son esenciales para el hogar. Este conocimiento resulta útil para predecir cuáles serán los efectos de un incremento en el flujo de ingresos entre las mujeres y entre los hombres (Kabeer, 2006).

A la hora de aplicar esta metodología para conceptualizar y medir la pobreza con una perspectiva de género, surge el problema de que, aunque las personas que colaboran sean tanto hombres como mujeres, la internalización de las normas de género en la comunidad puede implicar que ambos grupos ocultan o minimizan el sesgo de género (Chant, 2003). Las percepciones de las personas pobres, a menudo, reflejan normas y valores que no dan ningún peso a las desigualdades de género ni a las violaciones de derechos humanos que sufren las mujeres. Las propias mujeres aceptan sin reservas un sistema de valores que supone el dominio de los hombres en la toma de decisiones como un hecho «natural» y no injusto. Este problema también afecta a investigadores y analistas a la hora de interpretar y agregar los datos. Dependiendo de las habilidades del grupo de investigación, incluso cuando las evaluaciones participativas generen información relevante desde el punto de vista del género, pueden ocurrir pérdidas a la hora de transmitir esa información (Kabeer, 2006).

\subsection{Enfoque de las capacidades y la pobreza humana}

Amartya Sen argumenta que la pobreza se puede definir en términos de fracaso de las capacidades básicas de la persona para alcanzar determinados niveles mínimamente aceptables. Al hablar de pobreza y de nivel de vida, no hay que centrarse ni en los bienes, ni en sus características, ni en la utilidad que generan, sino en algo que él llama la capacidad de la persona. El bien o sus características, no nos dicen lo que la persona puede hacer con él; puede contribuir al nivel de vida, pero no es una parte constituyente de él (Sen, 1983, 1992a y 1992b). Lo importante es conocer la habilidad para hacer

5 Sobre la violencia como factor de empobrecimiento resulta interesante consultar Espinar (2002). 
cosas variadas usando ese bien, para conseguir determinados funcionamientos, y esta habilidad sería lo definido como capacidad. Con esta conceptualización, el marco analítico se enriquece puesto que la pobreza puede ser vista no sólo como una privación de recursos o medios para satisfacer las necesidades, sino también como una privación con respecto a los fines básicos o necesidades.

En el concepto de pobreza como fracaso de capacidades, cabe señalar dos rasgos que posibilitan la integración de la perspectiva de género en el estudio de la pobreza:

Pone de manifiesto que, incluso desde el estrecho punto de vista de los recursos, si no se toman en consideración una serie de heterogeneidades, la insuficiencia de recursos no captará ni siquiera una parte del problema de la pobreza. Tales heterogeneidades hacen que las necesidades no sean iguales para todos. Tienen que ver con el medio ambiente, el clima social, las relaciones sociales, las habilidades y circunstancias personales y el estado de salud. Un nivel de recursos dado no implica evitar la pobreza, entendida ésta como capacidad para alcanzar logros y funcionamientos, si una persona sufre violencia doméstica o si carece de capacidad para influir en la gestión del presupuesto del hogar. Las relaciones de género, por tanto, condicionan la situación de bienestar o pobreza.

La privación y la pobreza se pueden dar en múltiples aspectos, ya que implican el fracaso de las capacidades para alcanzar determinados funcionamientos, que van desde los físicamente elementales (como estar vestido y nutrido adecuadamente) hasta otros socialmente más complejos (como participar en la vida de la comunidad). Este punto de vista es compatible con la perspectiva de género, puesto que considera a mujeres y hombres no sólo como trabajadores y productores, sino también como actores que desempeñan múltiples roles en el hogar, en el trabajo y en la sociedad.

Sobre la base de las aportaciones de Sen, el PNUD ha desarrollado la noción de «pobreza humana», que se refiere a la incapacidad para lograr desarrollo humano alguno. La pobreza humana es calificada como una pobreza de opciones y oportunidades, que es más paralizante que la pobreza de ingresos. Significa entonces que se niegan las oportunidades y las opciones más fundamentales del desarrollo humano, es la ausencia de ciertas capacidades básicas para funcionar física y socialmente. Se entiende el desarrollo humano como un proceso de ampliación de las opciones de la gente, así como de elevación del bienestar logrado, que incluye vivir una vida larga y sana, tener educación y disfrutar de un nivel decente de vida y otras opciones adicionales, como la libertad política, la garantía de otros derechos humanos y diversos ingredientes del respeto por sí mismo.

Todas estas aportaciones han dado importantes frutos en cuanto a la incorporación del género en la medición de la pobreza. El Índice de Desarrollo relativo al Género (IDG) y el Índice de Potenciación del Género (IPG) miden el impacto de la inequidad 
de género en el desarrollo humano (IDG) y en la capacidad de acción (IPG) ${ }^{6}$. Aunque todavía hay limitaciones en cuanto a las estadísticas sobre las que se basan los índices, éstos son instrumentos complementarios importantes para estudiar las brechas de género y llaman la atención de los gobiernos, lo que influirá en las medidas para combatir la desigualdad de género. Hay que destacar que estos índices también han revelado las diferencias regionales de la desigualdad de género, que no coinciden necesariamente con las de pobreza, debido a las diferentes relaciones de parentesco y de género (Chant, 2003a: 30).

\subsection{Enfoque de la exclusión social}

El término «exclusión social» surge a finales de la década de 1980 para tratar de distinguir la exclusión social de otros viejos conceptos, como pobreza y privación; acentuar los aspectos dinámicos que están en la base de la pobreza, y producir un concepto menos unidimensional en relación con la pobreza financiera (Berghman, 1995: 16). $\mathrm{Su}$ creciente popularidad constituye un intento por entender e interpretar los nuevos modelos de división social que surgen a finales del siglo $\mathrm{xx}$, particularmente en relación con el cambio de los modelos empleo/desempleo, las modificaciones en la provisión del estado de bienestar, los cambios en los modelos de movilidad geográfica y en la definición de elegibilidad para una variedad de derechos y obligaciones civiles. Se identifican una serie de tendencias emergentes que tienen que ver con el crecimiento de la exclusión social, como las elevadas tasas de desempleo y subempleo, la absoluta y relativa disminución en la disponibilidad de trabajo manual y el crecimiento del trabajo de cuello blanco, el incremento de la participación de las mujeres en el trabajo remunerado, la introducción de innovaciones tecnológicas más sofisticadas dentro y fuera del trabajo, etc. (Littlewood y Herkommer, 1999: 2).

La pobreza se puede identificar más con aspectos de distribución y, en cambio, la exclusión social hace referencia a aspectos relacionales, a la inadecuación de la participación social, a la falta de integración social, o de poder, o a la negación de los derechos sociales (Room, 1995). No es simplemente pobreza económica; ni siquiera se refiere a la pobreza económica más extrema. Es una acumulación de barreras y límites que dejan fuera de la participación en la vida social mayoritaria a quienes la padecen.

Uno de los criterios que se han señalado como importantes para que un concepto sirva para analizar la situación de las mujeres y la naturaleza de la desigualdad de géne-

6 El IDG, al igual que el IDH (Índice de Desarrollo Humano), se basa en indicadores que consideran tres dimensiones -el estado de salud, el conocimiento y el nivel de vida- y se calcula con datos disociados para apreciar las disparidades por género. El IPG mide la equidad de género en términos de oportunidades económicas y políticas mediante cuatro indicadores: porcentaje de mujeres en escaños parlamentarios, porcentaje de mujeres legisladoras, oficiales superiores y gerentes, porcentaje de mujeres profesionales y trabajadoras técnicas, y relación del ingreso estimado entre hombre y mujeres. 
ro es que sea capaz de tomar una perspectiva comprensiva. Desde este punto de vista, el concepto de exclusión social posee una notable fortaleza, puesto que reconoce y valora diversas esferas de la vida aparte de la estrecha esfera de lo económico, y apunta hacia la interdependencia de procesos y relaciones entre esferas. El pensamiento feminista se ha movido en la misma dirección que el discurso de la exclusión social basado en el paradigma del monopolio ${ }^{7}$. Esto ha cambiado la atención desde el papel de la economía y los aspectos de distribución hacia la esfera simbólica y cultural de las relaciones de poder (Daly y Saraceno, 2002: 95-96). Además, el concepto de exclusión social ofrece un marco para enriquecer la investigación sobre la desventaja social porque sitúa los recursos materiales en un contexto (relacional, institucional, cultural y espacial), con lo que se pueden explorar las áreas de oportunidades y expectativas de las personas, aspecto fundamental a la hora de incorporar la perspectiva de género.

A partir de la Cumbre Mundial sobre el Desarrollo Social y la Cuarta Conferencia Mundial sobre la Mujer de 1995, se plantea la necesidad de abordar con herramientas conceptuales claras los mecanismos complejos que explican la persistencia y la magnitud de la pobreza de las mujeres. El concepto de exclusión social (que en la década de 1990 traspasa definitivamente las fronteras de Europa continental y se introduce en el discurso oficial internacional de lucha contra la pobreza) contiene importantes elementos que enriquecen mutuamente las perspectivas de exclusión y género. Entre ellos, destacan (Clert, 1998):

La incorporación de los aspectos socioculturales y políticos, además de los materiales. La dimensión sociocultural abarca el tema de la participación en las redes sociales y también aspectos relativos a la relación de las personas con las instituciones y a la no pertenencia a la cultura dominante. La dimensión política comprende la desigualdad de los miembros de una sociedad, que incluye los derechos civiles, políticos y sociales. La incorporación de ambas dimensiones permite apreciar las interrelaciones entre la pobreza y la falta de participación o de reconocimiento institucional o de derechos.

Pero, además, y como más novedoso, hay que mencionar que la perspectiva de la exclusión social conduce a análisis no estáticos, sino dinámicos; es decir, que tienen en cuenta los procesos y las prácticas de exclusión ejercidas por las diferentes instituciones, mercados y actores, y también el papel que cumple el espacio en los procesos de exclusión. El enfoque de exclusión social tiene potencialidad para examinar cómo los mercados, las instituciones, las empresas, el Estado y el territorio generan procesos de exclusión entre las mujeres.

7 Se trata de un paradigma en el que se considera que para luchar contra la pobreza se debe redistribuir, además del bienestar, el poder. Para más detalles sobre los tres paradigmas de la exclusión social (monopolio, especialización e integración), ver Silver (1995) y Levitas (1998). 
El análisis de la desventaja femenina bajo un enfoque multidimensional como el de exclusión social, que considera aspectos socioculturales y políticos, así como los mecanismos de exclusión generados por instituciones, actores, mercados y espacios, implica una metodología que va más allá de constatar que las mujeres tienen menos renta que los hombres, ya que pone de manifiesto los mecanismos y procesos mediante los cuales éstas llegan a una situación de múltiples privaciones y desventajas con respecto a los hombres.

El enfoque de la exclusión social es útil para analizar las relaciones de género porque no considera sólo las relaciones verticales, sino también las horizontales. Permite apreciar mejor cómo el poder no es sólo un asunto de grado, sino también de posición en una estructura de relaciones, y cómo las diferencias (en comportamiento, en atributos personales y en estilo de vida) cristalizan y se convierten en desigualdades. Así, la exclusión social permite ver más allá de la separación de actores y de esferas en los procesos de inclusión y exclusión, así como reconocer las interdependencias (Daly y Saraceno, 2002: 97).

En cuanto a las limitaciones que presenta el discurso de la exclusión social desde el punto de vista del género, cabe destacar dos (Daly y Saraceno, 2002: 97-99):

La importancia que se concede a los lazos y las obligaciones familiares es diferente para los hombres y para las mujeres. La mera pertenencia a la familia no se ve como un medio para conseguir la participación social, y esto se aplica a las madres solas. En cambio, sí que lo es tener un empleo remunerado aunque no se tengan lazos familiares, lo que se aplica a los hombres. Hay, por tanto, una jerarquía en las esferas. La de la familia, por sí sola, no es suficiente para lograr la integración social, pero la del trabajo remunerado sí.

En ninguno de los discursos de la exclusión social (ni en el francés de la solidaridad, ni en el anglo-norteamericano de la especialización, ni en el del monopolio) se le da un papel central a la actividad del cuidado y al trabajo no remunerado ${ }^{8}$. Es más, en el discurso anglo-norteamericano, como se restringe la inclusión a la integración en el mercado laboral, no sólo se infravalora el trabajo no remunerado y de cuidados, sino que también se margina.

8 Aunque no le da un papel central al trabajo no remunerado, el paradigma del monopolio, al menos, sí que reconoce este trabajo, y además contempla la integración social más allá de la participación laboral. El paradigma de la solidaridad reconoce el trabajo no remunerado sólo parcialmente, y contempla la integración social a través de su conversión en empleo remunerado. 


\section{Un primer examen de la sensibilidad hacia el género de algunas estadísticas y estudios sobre la pobreza y la exclusión social}

El método de cuantificación de la pobreza está, desde luego, relacionado con el enfoque y la definición de pobreza que se tome como punto de partida. La metodología de cuantificación de la desventaja social ha conocido importantes avances, que se han plasmado en la elaboración de indicadores más precisos y en la incorporación de un mayor número de dimensiones, avances que están relacionados con los habidos en el ámbito teórico. Sin embargo, no se puede decir que haya habido una evolución paralela de conceptos y mediciones. La riqueza de los conceptos no siempre suele plasmarse en estudios aplicados, debido a las dificultades para hacer operativos tales conceptos. Así que puede decirse que, en el campo de la medición, con respecto al de la conceptualización, se lleva cierto retraso.

Como ya decíamos en páginas anteriores, los enfoques que van más allá de lo económico y que consideran las diferentes esferas de la vida (como el de capacidades o el de exclusión social) van a presentar mayor potencialidad de cara a realizar una medición de la pobreza sensible al género. Sin embargo, el todavía escaso desarrollo de mediciones de la pobreza y la exclusión social con perspectiva multidimensional es un obstáculo para introducir el enfoque de género los trabajos empíricos. Así, son las mediciones convencionales (método indirecto basado en la renta o el gasto) las que más abundan. En algunas se incorporan algunos aspectos interesantes desde la óptica del género, mientras que otras no van más allá de tratar la variable sexo como una más (edad, estado civil, nivel educativo, etc.). A continuación, vamos a referirnos a algunos de estos trabajos empíricos, destacando sus carencias e innovaciones desde la perspectiva de género. No pretendemos ser exhaustivos, es decir, no vamos a referirnos a todos los trabajos y análisis realizados al respecto, sino a los realizados por algunos organismos oficiales e institutos estadísticos, y que hemos manejado en trabajos anteriores, por lo que estamos familiarizados con sus metodologías y resultados. No significa, por tanto, que no haya otros trabajos relevantes en este campo, además de los mencionados en estas páginas.

Las estadisticas de Eurostat sobre renta, pobreza y exclusión social (2002) sí que ofrecen datos por sexo a la hora de cuantificar la pobreza y la privación social, aunque no manejan un concepto sensible al género. A la hora de estudiar la exclusión social, se incorporan dimensiones relevantes de cara a estudiar las diferencias entre la desventaja social femenina y la masculina, como son las relaciones sociales, la salud o la percepción subjetiva de las circunstancias económicas. Sin embargo, la ausencia de datos disociados por sexo sobre exclusión social impide un análisis que podría resultar 
fructífero para el diseño de políticas. Otro tanto ocurre con el estudio del papel de las transferencias sociales en la reducción de la tasa de pobreza. Su desagregación por sexo permitiría poner de manifiesto las desigualdades entre mujeres y hombres en el acceso a los sistemas de protección social y, por tanto, impulsar reformas en los mismos para combatir la pobreza con más eficacia.

La Encuesta de Condiciones de Vida que se realiza desde 2007 en la UE, además del estudio de la renta, abarca también las condiciones de trabajo, de la vivienda, el nivel formativo, el estado de salud y aspectos referentes al cuidado de hijos. Contiene, por tanto, un volumen de información muy relevante para el estudio de la desventaja social con una perspectiva pluridimensional. Sin embargo, como cada dimensión se trata de manera independiente con respecto a las demás, no es posible extraer datos que relacionen diferentes dimensiones e indiquen cuántas personas y de qué características sufren diversas privaciones simultáneamente. Tampoco hay datos disociados por sexo de cada una de las dimensiones y subdimensiones estudiadas, ni cruces de la variable sexo con otras como la edad, el nivel formativo o la situación laboral. Podemos decir, por tanto, que esta encuesta es muy mejorable desde la óptica de género.

La Encuesta sobre las Personas sin Hogar (2005) realizada por el INE tiene mayor sensibilidad hacia el género que las anteriores, puesto que ofrece datos disociados por sexo de todas y cada una de las variables estudiadas (edad, lugar de nacimiento, situación familiar, nacionalidad, situación laboral, nivel de ingresos, nivel de estudios, etc.). Esto representa un importante avance, ya que permite apreciar las diferencias en la problemática del sinhogarismo entre mujeres y hombres. Así mismo, como la dimensión residencial se trata en relación con otras como la educativa, la laboral, la de la salud, la del ingreso, etc., posee un buen potencial para comprender los procesos complejos y diversos que llevan a mujeres y hombres a situaciones de exclusión tan extremas. También debe señalarse lo interesante que resulta que en esta encuesta se haya estudiado la situación familiar, cuyas diferencias entre mujeres y hombres sin hogar son importantes y nos indican el diferente peso que alcanza la problemática de la soledad entre ellas y ellos ${ }^{9}$. Entre las posibles mejoras, hay que mencionar que la encuesta no trata el tema de la convivencia con hijos entre las personas sin hogar. Pues bien, resultaría muy revelador hacerlo, ya que una parte significativa de las mujeres sin hogar tiene hijos a su cargo (se habla en este caso de familias sin hogar) y se encuentra con la problemática de que pocos centros atienden a familias.

La Encuesta de Pobreza y Desigualdades Sociales (EPDS) que se realiza en la Comunidad Autónoma del País Vasco desde $1986^{10}$ tiene una base metodológica convencional, puesto que el núcleo fundamental de la medición de las desigualdades y de la

9 Entre ellas, casi el 69\% vive en pareja, mientras que entre ellos sólo el 26,6\%.

10 Se han realizado 5 encuestas: 1986, 1996, 2000, 2004 y 2008. 
pobreza se centra en los recursos monetarios de los que dispone la persona o el hogar. Ahora bien, la encuesta introduce una serie de elementos que enriquecen de manera importante esta metodología y proporcionan una valiosa información complementaria (De la Cal, 2007).

Cuando estudiábamos el enfoque monetario decíamos que uno de los obstáculos que presentaba era que se solía cuantificar la pobreza sólo por hogares y no por personas, lo que ocultaba situaciones de vulnerabilidad de colectivos como la juventud no emancipada, las personas mayores y las mujeres que no realizan trabajo remunerado. Para evitar esto, además de ofrecer los datos tanto por hogares como por personas, la EPDS incorpora un epígrafe en el que se valora la situación de pobreza o bienestar de la persona en función de sus ingresos estrictamente al margen de los del resto de las personas que integran el hogar. De esta forma se evalúa la capacidad de cada persona para acceder a un modo de vida independiente. Este aspecto resulta muy revelador de cara a incorporar la perspectiva de género en el estudio de la pobreza, puesto que nos da idea de la verdadera capacidad de las mujeres a la hora de tener una vida autónoma.

Los resultados obtenidos para el año 2008 son bastante llamativos, puesto que se estima que entre las personas mayores de 18 años (excluidos los estudiantes entre 18 y 25 ) casi un $30 \%$ no dispone de recursos suficientes para vivir independientemente; es decir, si se independizase, sufriría pobreza. En el caso de las mujeres, el porcentaje es muy superior: un 47,1\% frente al 10,7\% de los hombres. Hay que destacar además que en el conjunto de la población que sería pobre si se independizase, el $82,9 \%$ son mujeres.

Por último, cabe señalar que las EPDS se aproximan a la problemática de falta de capacidad de las mujeres para acceder y administrar los recursos del hogar de manera que se satisfagan las necesidades de todos sus componentes. Así, se ha introducido una pregunta exploratoria para aproximarse a la incidencia de las problemáticas de acceso no compartido a los recursos. Para ello se han utilizado las respuestas correspondientes a mujeres que viven en pareja, adscritas al grupo principal del hogar e informantes directas en el proceso de encuesta. Resulta significativo que el acceso no compartido a los recursos existe en un 1,8\% de las personas mencionadas, y que la problemática es más acentuada cuanto peor es la situación económica (el porcentaje sube hasta el 6,5\% entre las mujeres que sufren pobreza).

Como aspectos mejorables en las EPDS, cabe mencionar la falta de desagregación por sexo de los indicadores de precariedad (que nos dan una aproximación a la exclusión social) y, al igual que en las demás encuestas que hemos citado, que no se ofrecen datos de edad, situación laboral, nivel formativo, etc., desagregados para mujeres y hombres. 


\section{Conclusiones}

El primer paso para que una problemática se aborde mediante las políticas públicas es que sea puesta de manifiesto y reconocida por la sociedad. Un enfoque de pobreza y exclusión social que sea sensible al género constituye el primer paso para diseñar y organizar las actuaciones y políticas para su reducción, dado que permite evidenciar la mayor incidencia e intensidad de la pobreza entre las mujeres, así como los procesos que conducen a ella.

Los enfoques multidimensionales, como el de capacidades o el de exclusión social, tienen más interés a la hora de incorporar la perspectiva de género, ya que consideran las diferentes esferas de la vida (hogar, trabajo y sociedad) y no sólo la económicomercantil propia del enfoque monetario. Con todo, en este último enfoque pueden introducirse cambios que mejoran significativamente su sensibilidad hacia el género.

En cuanto a las estadísticas y análisis sobre la pobreza y la exclusión social, es necesario trascender la mera presencia del sexo como una variable más e incorporarlo como categoría transversal, de manera que cualquier información relativa a las personas que se ofrezca esté desdoblada por sexo.

Estudiar, analizar y medir las desventajas que sufren muchas mujeres en nuestros sistemas sin tomar en consideración lo expuesto, llevará a que las políticas de lucha contra la pobreza simplemente alivien las privaciones, pero sin que haya una modificación sustancial del statu quo. La intervención con vocación transformadora exige incorporar la perspectiva de género para remover los obstáculos que impiden que las mujeres participen en pie de igualdad con los hombres en las diferentes esferas y, además, para que cada una de estas esferas sea considerada igualmente valiosa. Ello ampliará las oportunidades y opciones de las mujeres, pero también las de los hombres, y servirá para reducir la pobreza humana.

\section{Bibliografía}

Alcock, P. (1997). Understanding poverty. London: Macmillan.

Arriagada, I. (2006). «Dimensiones de la pobreza y políticas desde una perspectiva de género», en Revista de la CEPAL, 85. httpः//www.eclac.org/publicaciones/ $\mathrm{xml} / 6 / 21046 / \operatorname{lcg} 2266 \mathrm{eArriagada} . \mathrm{pdf}$

Berghman, J. (1995). "Social exclusion in Europe: policy context and analytical framework», en Room, G. (ed.), Beyond the threshold. London: The Policy Press. 
Bravo, R. (1998). «Pobreza por razones de género. Precisando conceptos», en Arriagada, I. y Torres, C. (comp.), Género y pobreza. Nuevas dimensiones. Santiago de Chile: ISIS Internacional.

Brunet, I.; Valls, F., y Belzunegui, A. (2008). «Pobreza, exclusión social y género», en Sistema, 207: 69-85.

CEPAL (2003). «Pobreza y desigualdad desde una perspectiva de género», en Panorama social de América Latina. Naciones Unidas.

CEPAL-UNIFEM (2004). «Entender la pobreza desde la perspectiva de género», en Serie Mujer y Desarrollo, n. ${ }^{\circ}$ 52. Unidad Mujer y Desarrollo.

CANT, S. (2003a). «Nuevas contribuciones al análisis de la pobreza: desafíos metodológicos y conceptuales para entender la pobreza desde una perspectiva de género», en Serie Mujer y Desarrollo, n. ${ }^{\circ}$ 47. Unidad Mujer y Desarrollo. CEPAL. http://www. eclac.cl/publicaciones/xml/7/14837/lcl1955e.pdf

Chant, S. (2003b). «Female household headships and the feminisation of poverty: facts, fictions and forward strategies», en Working Paper, 9. London School of Economics, Gender Institute.

Clert, C. (1998). «De la vulnerabilidad a la exclusión: género y conceptos de desventaja social», en Arriagada, I. y Torres, C. (comp.), Género y pobreza. Nuevas dimensiones. ISIS Internacional. Santiago de Chile.

Concialdi, P. (2002). «Les seuils de pauvreté monétaire: usages et mesures», en Revue de l'IRES, 38: 109-136.

Daly, M. y Saraceno, C. (2002). «Social exclusion and gender relations», en Hobson, B.; Lewis, J. y Sirm, B. (ed.), Contested concepts in gender and social politics. Reino Unido: Edward Elgar.

De La CaL, M. L. (2003). Cambios laborales y nueva pobreza en los países desarrollados: algunas propuestas preventivas. Tesis doctoral. UPV/EHU.

De La Cal, M. L. (2007). «Luces y sombras en las Encuestas de Pobreza y Desigualdades Sociales desde el punto de vista del género», en Zerbitzuan, 42: 19-28.

Espinar, E. (2002). «La violencia doméstica como factor de empobrecimiento», en Tortosa, J. M. (coord.), Mujeres pobres, indicadores de empobrecimiento en la España de hoy. Fundación Foessa.

EUROSTAT (2002). European social statistics. Income, poverty and social exclusion. $2^{\text {nd }}$ report (1994-1997). Luxemburgo: Comisión Europea.

Gobierno Vasco-Eusko Jaurlaritza (1997, 2000 y 2004). Encuestas de pobreza y desigualdades sociales. Vitoria-Gasteiz: Departamento de Justicia, Economía, Trabajo y Seguridad Social. 
Gobierno Vasco-Eusko Jaurlaritza (2008). 25 años de estudio de la pobreza en Euskadi (1984-2008). Vitoria-Gasteiz: Departamento de Justicia, Empleo y Seguridad Social.

KABEER, N. (1998), «Tácticas y compromisos: nexos entre género y pobreza», en Arriagada, I. y Torres, C. (comp.), Género y pobreza. Nuevas dimensiones. Santiago de Chile: ISIS Internacional.

KABEER, N. (2006). Lugar preponderante del género en la erradicación de la pobreza y las metas del desarrollo del milenio. Plaza y Valdés. http://www.idrc.ca/openebooks/172-8/

Levitas, R. (1998). The inclusive society? Social exclusion and new labour. Londres: Macmillan Press.

Littlewood, P. y Herkommer, S. (1999). »Identifying social exclusion», en Littlewood, P. (ed.), Social Exclusion in Europe. England: Ashgate.

Martínez, A. (2001). «Género, pobreza y exclusión social: diferentes conceptualizaciones y políticas públicas», en Tortosa, J. M. (coord.), Pobreza y perspectiva de género. Barcelona: Icaria.

Mateo, M. A. (2001). «Desigualdad, pobreza y exclusión: conceptos, medidas y alternativas metodológicas», en ToRTOSA, J. M. (coord.), Pobreza y perspectiva de género. Barcelona: Icaria.

Monreal, P. (1999). "iSirve para algo el concepto de cultura de la pobreza?», en Revista de Occidente, 216: 75-88.

Munk, M.D. (2002). Gender, marginalisation and social exclusion. Working Paper 14/a. The Danish National Institute of Social Research.

Room, G. (1995). «Poverty and social exclusion», en Room, G. (ed.). Beyond the threshold. The Policy Press. University of Bristol.

Sen, A. (1983). "Poor, relatively speaking". Oxford Economic Papers, 35: 153-169.

Sen, A. (1992a). Nuevo examen de la desigualdad. Madrid: Alianza.

SEN, A. (1992b). «Sobre conceptos y medidas de la pobreza», en Comercio Exterior, 42, (4): 310-322.

Sen, G. (1998). «El empoderamiento como enfoque de la pobreza», en ArriagaDA, I. y Torres, C. (comp.), Género y pobreza. Nuevas dimensiones. Santiago de Chile: ISIS Internacional.

Silver, H. (1995). «Fighting social exclusion», en Social Exclusion, social inclusion. Belfast. Democratic Dialogue. http://cain.ulst.ac.uk/dd/report2/report2.htm

Tortosa, J. M. (2002). Mujeres pobres, indicadores de empobrecimiento en la España de hoy. Fundación Foessa.

U.S. Census Bureau (2008). Income, poverty and health insurance coverage in the United States: 2007. U.S. Departament of Commerce. 